\title{
Prostaglandin Receptor EP4 in Abdominal Aortic Aneurysms
}

\author{
Richard Y. Cao, ${ }^{*}$ Tim St. Amand, ${ }^{*}$ XinZhi Li, ${ }^{*}$ \\ Sung-Hee Yoon, ${ }^{*}$ Carol P. Wang, ${ }^{*}$ Hui Song, ${ }^{\dagger}$ \\ Takayuki Maruyama, ${ }^{\ddagger}$ Peter M. Brown, ${ }^{\S}$ \\ David T. Zelt, ${ }^{\S}$ and Colin D. Funk* \\ From the Departments of Biomedical and Molecular Sciences," \\ Mathematics and Statistics, ${ }^{\dagger}$ and Vascular Surgery, ${ }^{\circledR}$ Queen's \\ University and Kingston General Hospital, Kingston, Ontario, \\ Canada; and the Development Research Laboratories, ${ }^{\ddagger}$ Research \\ Headquarters, Ono Pharmaceutical Co., Ltd., Osaka, Japan
}

Abdominal aortic aneurysm (AAA) pathogenesis is distinguished by vessel wall inflammation. Cyclooxygenase (COX)-2 and microsomal prostaglandin E synthase-1, key components of the most well-characterized inflammatory prostaglandin pathway, contribute to AAA development in the 28-day angiotensin II infusion model in mice. In this study, we used this model to examine the role of the prostaglandin $\mathrm{E}$ receptor subtype 4 (EP4) and genetic knockdown of COX-2 expression (70\% to $90 \%$ ) in AAA pathogenesis. The administration of the prostaglandin receptor EP4 antagonist AE3-208 $(10 \mathrm{mg} / \mathrm{kg}$ per day) to apolipoprotein $\mathrm{E}$ (apoE)-deficient mice led to active drug plasma concentrations and reduced AAA incidence and severity compared with control apoE-deficient mice $(P<$ 0.01), whereas COX-2 genetic knockdown/apoE-deficient mice displayed only a minor, nonsignificant decrease in incidence of AAA. EP4 receptor protein was present in human and mouse AAA, as observed by using Western blot analysis. Aortas from AE3-208treated mice displayed evidence of a reduced inflammatory phenotype compared with controls. Atherosclerotic lesion size at the aortic root was similar between all groups. In conclusion, the prostaglandin $E_{2}-$ EP4 signaling pathway plays a role in the AAA inflammatory process. Blocking the EP4 receptor pharmacologically reduces both the incidence and severity of AAA in the angiotensin II mouse model, potentially via attenuation of cytokine/chemokine synthesis and the reduction of matrix metalloproteinase activities. (Am J Pathol 2012, 181:313-321; bttp://dx. doi.org/10.1016/j.ajpath.2012.03.016)
Abdominal aortic aneurysm (AAA), characterized by a dilatation exceeding the normal diameter by $>50 \%,{ }^{1}$ is associated with advanced age, male sex, cigarette smoking, atherosclerosis, hypertension, and genetic predisposition. ${ }^{2-4}$ The histopathological features of AAAs are characterized by chronic inflammatory cell recruitment to the aortic wall, with tissue degeneration and remodeling, and depletion of medial smooth muscle cells. ${ }^{5,6}$ AAAs are a common vascular condition with life-threatening implications from aortic rupture, which has been reported to have a mortality rate as high as $90 \%{ }^{2}$ and causes $>15,000$ deaths per year in the United States. ${ }^{7}$ Surgical repair by standard means or interventional endovascular stent placement is the only option for treatment. Therefore, developing pharmacological prevention strategies to block AAA progression is a high priority.

Cyclooxygenase (COX)-2, an enzyme that generates inflammatory mediators, such as prostaglandin $E_{2}\left(P E_{2}\right)$, was highly expressed in human AAA specimens, and the enzyme was proposed as a potential target for pharmacotherapy. ${ }^{8}$ To test this assertion in animals, experiments involving both genetic and pharmacological inhibition of COX-2 were conducted using the well-characterized angiotensin II (Angll)-induced mouse AAA model. Results revealed that COX-2 contributed significantly to AAA formation. ${ }^{9,10}$ Because of the potential renal and cardiovascular risks of COX-2 selective inhibition, ${ }^{11-14}$ downstream blockade in the $\mathrm{PGE}_{2}$ arm of the pathway was tested using mice with disruption of microsomal prostaglandin E synthase-1 (mPGES-1) expression. This resulted in suppressed AAA formation in the Angll model with mice on a low-density lipoprotein receptor-deficient background. ${ }^{15}$ mRNA-en-

Supported by a grant from the Canadian Institutes of Health Research (MOP-93689 to C.D.F.). C.D.F. is the recipient of a Tier I Canada Research Chair in Molecular, Cellular and Physiological Medicine and is a Career Investigator of the Heart and Stroke Foundation of Ontario.

Accepted for publication March 6, 2012.

Disclosures: T.M. is employed by Ono Pharmaceutical Co., Ltd., which supplied the EP4 antagonist, AE3-208, used in these studies.

Supplemental material for this article can be found on http://ajp. amjpathol.org or at $h$ ttp://dx.doi.org/10.1016/j.ajpath.2012.03.016.

Address reprint requests to Colin D. Funk, Ph.D., Department of Biomedical and Molecular Sciences, Botterell Hall Room 433, Queen's University, Kingston, Ontario, Canada K7L 3N6. E-mail: funkc@queensu.ca. 
coding $\mathrm{PGE}_{2}$ receptor subtypes EP2, EP3, and EP4 have been detected in human AAA specimens, and parallel in vitro experiments demonstrated that $\mathrm{PGE}_{2}$ stimulated secretion of IL-6 from aortic macrophages. ${ }^{16}$ Taken together, results from these studies implicate a COX-2/mPGES-1/EP receptor pathway potentially linked via cytokines/chemokines, as suggested by others, ${ }^{17-19}$ to inflammatory circuits in AAA formation.

The purposes of the present study were to study the role of one EP receptor subtype, EP4, in this AAA signaling axis using a selective EP4 antagonist in the Ang II AAA murine model; to examine if EP4 receptor protein is present in human AAA; and to determine if genetic COX-2 knockdown (COX2KD) differs from COX-2 knockout in terms of phenotype in the murine model. Because $\mathrm{PGE}_{2-}$ EP4 signaling promotes type 17 helper T-cell expansion and IL-17 production, ${ }^{20,21}$ a pleiotropic cytokine, which mediates pro-inflammatory responses, we also investigated the presence of IL-17 in AAA samples and other aspects of the inflammatory process.

\section{Materials and Methods}

\section{Mouse AAA Model Induction and Drug Intervention}

The Angll-induced mouse AAA model, using an atherosclerotic-susceptible strain [either apolipoprotein E deficient $\left(\mathrm{apoE}^{-1-}\right)$ or low-density lipoprotein receptor deficient], has become an exceedingly popular tool because of its simplicity and because certain facets of the model resemble human disease acquisition, including male sex preponderance in the setting of mild hypertension with enhanced incidence in the presence of hyperlipidemia. $^{22,23}$ In this study, apoE ${ }^{-1-}$ mice on a C57BL/6 genetic background (Jackson Laboratory, Bar Harbor, $\mathrm{ME})$ were crossbred with COX2KD mice in which COX-2 expression was reduced by $70 \%$ to $90 \%$, but not eliminated. ${ }^{24}$ Alzet osmotic minipumps (model 2004; Durect Corporation, Cupertino, CA), loaded with Angll (SigmaAldrich, St Louis, MO), were implanted s.c. into 3-monthold chow-fed male mice in the dorsal region under isoflurane anesthesia (delivered in $100 \% \mathrm{O}_{2}$ ) to obtain a delivery rate of $1 \mu \mathrm{g} / \mathrm{kg}$ per minute during the course of 4 weeks, as previously described. ${ }^{25}$ The plane of anesthesia was monitored by lack of response to toe pinch and even respiration rate. Mice with comparable body weights were divided into three groups: group 1, control $\left(\right.$ apoE ${ }^{-1-}$ ); group 2, COX2KD on an apoE ${ }^{-1-}$ background; and group 3, EP4 antagonist-treated apoE ${ }^{-1-}$ (Table 1). The EP4 antagonist, ONO-AE3-208 (Ono Pharmaceutical Co, Ltd, Osaka, Japan), was administered via

Table 1. Mice in the Study Groups

\begin{tabular}{|c|c|c|c|}
\hline Groups & Genotypes & Treatment & Body weight $(\mathrm{g})^{*}$ \\
\hline Control & $a p o E^{-1-}$ & Angll & $31.5 \pm 0.5(n=31)$ \\
\hline COX2KD & COX2KD/apoE $E^{-\prime-}$ & Angll & $32.3 \pm 0.7(n=27)$ \\
\hline AE3-208 & $a p o E^{-1-}$ & Angll + AE3-208 & $31.0 \pm 0.7(n=23)$ \\
\hline
\end{tabular}

$P>0.05$ for control versus either COX2KD or AE3-208.

${ }^{*}$ Data are given as mean \pm SEM. the drinking water at $10 \mathrm{mg} / \mathrm{kg}$ per day, starting 1 week before Angll infusion and for the complete 4-week duration of Angll infusion. Fresh water with drug was replaced at weekly intervals. The Animal Use Committee at Queen's University (Kingston, ON, Canada) approved the animal protocols described herein (Funk-2009-086), and experiments conform to the $\mathrm{NIH}$ guidelines.

\section{Ultrasonographic Imaging Acquisition}

Abdominal ultrasonographic imaging was performed under isoflurane anesthesia using a Vevo 770 high-resolution ultrasound system (VisualSonics, Toronto, ON) with a $40-\mathrm{MHz}$ frequency real-time microvisualization scan head (RMV 704) and a $10 \times 10-\mathrm{mm}$ field of view in two-dimensional and three-dimensional (3D) modes. This method was used to monitor AAA progression at 1 and 2 weeks after Angll infusion initiation and just before euthanasia by $\mathrm{CO}_{2}$ asphyxiation at 4 weeks. The 3D data were further reconstructed into volume measurements using VisualSonics software version 3.0 (Vevo 770; Toronto, ON, Canada).

\section{AAA Assessment}

After mice were euthanized by $\mathrm{CO}_{2}$ asphyxiation on day 28 of Angll infusion, blood was collected from the inferior vena cava for drug concentration and lipid profile analysis. Hearts were prepared for aortic root atherosclerotic lesion analysis, and aortic trees from the distal iliac bifurcation to the proximal aortic root were carefully dissected free from surrounding tissue. Abdominal aortas with aneurysms were measured at the greatest suprarenal diameter, whereas aortas without aneurysms were measured approximately 2 $\mathrm{mm}$ above the right renal artery, where most AAAs develop; they were measured with a micrometer and scored according to a previous classification. ${ }^{26}$ These specimens were embedded in optimal cutting temperature medium and stored at $-80^{\circ} \mathrm{C}$ until sections were cut for further histological and IHC analysis. Some mice did not have the AAA diameter measured because of aortic rupture and other reasons (eg, a mouse died 2 days before end point because of unidentified reasons or nonrupture related).

\section{Histological and Immunofluorescence Staining}

AAA sections were stained with Movat's pentachrome for morphological analysis. CD90.2, CD4, CD68, CD80, CD163, interferon- $\gamma$, IL-2, IL-17, and macrophage inflammatory protein (MIP)- $1 \alpha$ expression levels were tested by immunofluorescence methods, as previously described. ${ }^{27}$ Briefly, AAA sections were fixed with acetone for 5 minutes, washed, blocked with $3 \%$ normal goat serum for 30 minutes and then incubated with primary antibodies against CD90.2 (BD Pharmingen, Mississauga, ON), CD163 (Santa Cruz Biotechnology, Santa Cruz, CA), CD68 and CD80 (Serotec, Oxford, UK), IL-17 (Epitomics, Burlingame, CA), and MIP- $1 \alpha$ (Novus Biologicals, Littleton, CO) for 2 hours. After washing with PBS, specimens were incubated with appropriate fluorescently labeled secondary antibodies (Jackson ImmunoResearch, West Grove, PA) for 1 hour. Coverslips were mounted with VECTASHIELD plus DAPI (Vector, Burlin- 
game, CA). Visualization was performed with a DM-IRB fluorescent microscope (Leica, Richmond Hill, ON). Quantification of positively stained cells was conducted with Image-Pro Plus software version 5.1 (Media Cybernetics, Silver Spring, MD), counting an equal number of defined fields on multiple slides between each of the three treatment groups/genotypes, summing the total positive cells, and generating a percentage (relative to DAPI-stained cells). Atherosclerotic lesions at the aortic root were quantified by counting the total lesion area stained by oil red O (Sigma-Aldrich).

\section{MMP Zymography Assay}

Aortic matrix metalloproteinase (MMP)-2 and MMP-9 activities were analyzed using gelatin zymography, as previously described. ${ }^{17}$ Briefly, aortic samples from control and AE3-208-treated mice were homogenized and equal amounts of protein (Bio-Rad protein assay; Bio-Rad, Mississauga, ON, Canada) were loaded into wells of a $10 \%$ SDS-polyacrylamide gel containing $0.1 \%$ gelatin and electrophoresed under nonreducing conditions. Samples from a mouse embryo fibroblast cell line (a generous gift from Dr. Alan Mak's laboratory, Department of Biomedical and Molecular Sciences, Queen's University) were loaded as positive control. After electrophoresis, the proteins were renatured by soaking the gel in renaturing buffer and then placed into developing buffer. Gels were stained with Coomassie Blue and then destained with acetic acid until areas of protease activity appeared as clear bands against a dark blue background, where proteases had digested the substrate.

\section{Analysis of ONO-AE3-208 Plasma Concentration}

The plasma concentrations of drug were analyzed at study end point by liquid chromatography-tandem mass spectrometry with multiple reaction monitoring in electrospray ionization-positive ion mode using a Prominence_XR (Shimadzu, Kyoto, Japan) high performance liquid chromatography/API4000 (AB Sciex Tokyo, Japan) mass spectrometer with a Shim-pack XR-ODSII analytical column (2.0-mm internal diameter $\times 75 \mathrm{~mm}$ length of column; Shimadzu). The mobile phase consisted of solvent $\mathrm{A}$ (water containing $0.1 \%$ formic acid) and solvent B (acetonitrile containing $0.1 \%$ formic acid) starting at a 9:1 ratio, with a gradient over 1.5 minutes to a 1:9 ratio, and constant at this ratio until 3 minutes, with a flow rate of $0.5 \mathrm{~mL} /$ minute. Candesartan was used as an internal standard.

\section{Human Aorta Samples and Processing}

Twenty-five AAA specimens from the infrarenal segment of the abdominal aorta in patients (17 males and 8 females; aged 57 to 84 years) undergoing surgery for AAA repair were obtained, along with three control nonaneurysm tissues ( 3 males; aged 43 to 48 years) removed from the infrarenal segment of the abdominal aorta postmortem (within 24 hours). Demographics are found in Supplemental Table S1 (available at http://ajp.amjpathol. org). All tissues were washed with one times PBS to remove blood, then snap frozen in liquid nitrogen and kept at $-80^{\circ} \mathrm{C}$. Tissues were obtained with approval from the Research Ethics Board of the Faculty of Health Sciences, Queen's University and Kingston General Hospital, and the investigation conforms to the principles outlined in the Declaration of Helsinki.

Pieces of aortic tissues were fixed in 10\% neutral-buffered formalin and embedded in paraffin. Sections $(8 \mu \mathrm{m}$ thick) were prepared, consisting of the entire thickness of the vessel wall and stained with modified Movat's pentachrome, and were subsequently mounted in Permount (Fisher Scientific, Fair Lawn, NJ) and coverslip protected. Additional pieces were resuspended in tissue protein extraction reagent (ThermoScientific, Rockford, IL) plus a protease inhibitor (Roche Diagnostics, Indianapolis, IN) mixture, followed by sonication on ice (three times, 5 seconds each). The sonicated homogenate was centrifuged at 13,000 $\times g$ for 5 minutes, and the supernatant fraction was assayed (Bradford method) to determine protein concentration.

Proteins $(30 \mu \mathrm{g})$ were subjected to $12 \%$ SDS-PAGE and transferred onto polyvinylidene difluoride membranes (Millipore Corporation, Billerica, MA) via a semidry transfer technique. The membranes were blocked overnight in 5\% milk (suspended in Tris-buffered saline) at $4^{\circ} \mathrm{C}$. Membranes were treated with an anti-human EP4 receptor polyclonal antibody (1:200 dilution; catalogue number sc20677; Santa Cruz Biotechnology, Santa Cruz, $\mathrm{CA}$ ) or anti- $\beta$-actin monoclonal antibody (1:5000 dilution; Sigma-Aldrich Co, Oakville, ON) for 1 hour at room temperature, followed by washing and incubation with the respective secondary antibodies (1:2000 anti-rabbit and 1:10,000 anti-mouse) in 5\% milk and Tris-buffered saline. Protein bands were visualized on a FluorChem 8900 instrument (Alpha Innotech, San Leandro, CA) after the application of a chemiluminescence detection reagent (GE Healthcare, Buckinghamshire, UK). ImageJ software version 1.45s $(\mathrm{NIH}$, Bethesda, MA) was used to quantify the protein band intensities. The intensities of the protein of interest were normalized to $\beta$-actin to generate an expression ratio.

\section{Statistical Analysis}

Data are expressed as mean \pm SEM. Differences between two groups were analyzed by the Student's t-test. Correlation was tested by linear regression and Pearson coefficient analyses. Differences of two proportions were analyzed by Fisher's exact test. The calculation of power for the detection of observed difference was performed using the formula of Rosner, ${ }^{28}$ which was based on the normal approximation for the distribution of the test statistics. $P<0.05$ was considered significant.

\section{Results}

Comparative Effects of Genetic COX2KD versus $P G E_{2}$ Receptor EP4 Antagonist Treatment on Angll-Induced AAA Incidence and Severity

This study was designed to test the impact of either genetic COX2KD or pharmacological inhibition of the $\mathrm{PGE}_{2}$ receptor EP4 on AAA pathogenesis using three 

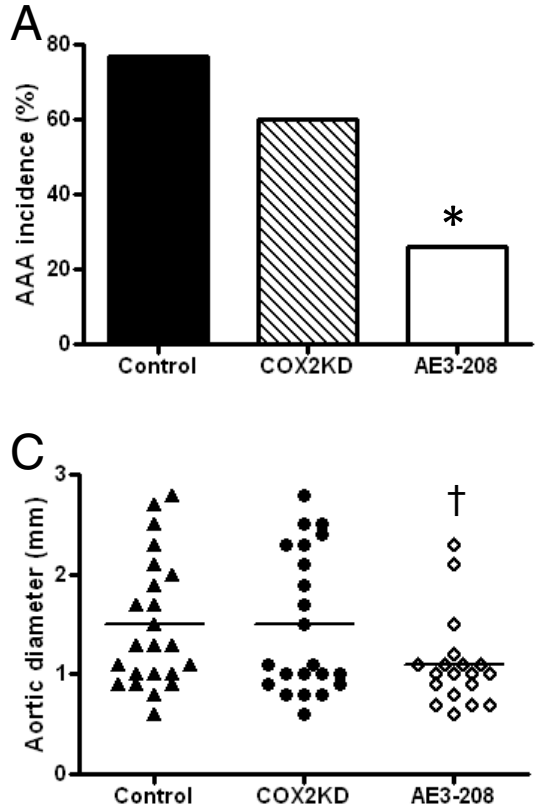

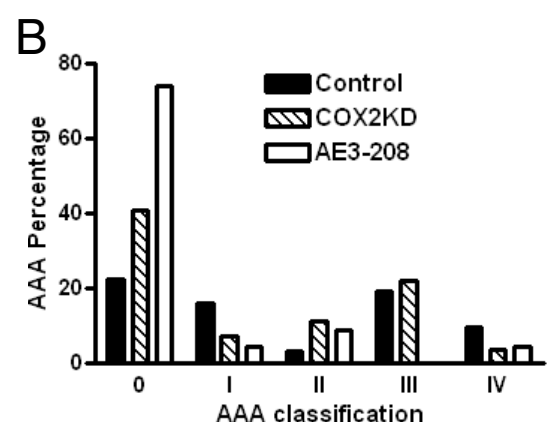

D

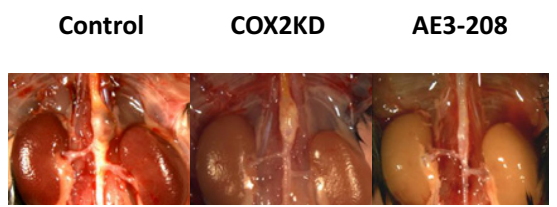

Figure 1. The EP4 antagonist AE3-208 decreases AAA incidence and severity in AngII-infused mice. A: EP4 antagonist treatment significantly decreases AAA incidence versus the control group (26\% versus $77 \%)$. ${ }^{*} P<0.01$. B: Mice are classified according to a scale previously described: 0 , no aneurysm; I, small aneurysm; II, midsized aneurysm; III midlarge aneurysm with thrombus; and IV, multiple aneurysms. EP4 antagonist-treated mice have no severe type III AAAs. Percentages do not total $100 \%$ because ruptures, which are classified as AAA, are not included. C: EP4 antagonist-treated mice display a significantly smaller average aortic diameter versus the control group of mice (1.1 \pm 0.10 versus $1.5 \pm 0.13 \mathrm{~mm}) .{ }^{\dagger} P<0.05$. D: Representative images of abdominal aorta in the three groups of mice [ $n=31, n=27$, and $n=23$ (A and B) and $n=23, n=22$, and $n=19(\mathbf{C})]$, respectively, for the control, COX2KD, and AE3-208 groups. groups of male apoE-deficient mice (Table 1). Abdominal aortic enlargements and aneurysm complications, such as aortic dissections and ruptures, were monitored by both ultrasonographic screening and end point dissection. Suspected abdominal aortic expansions $>50 \%$ of the original aortic size were considered as aortic aneurysms. COX2KD mice had a lower AAA incidence com- pared with control mice [16 (59\%) of 27 versus 24 (77\%) of 31], which did not reach statistical significance (Figure 1A). AAA severity based on the classification of Daugherty et $\mathrm{al}^{23}$ and aortic diameter $(1.5 \pm 0.15$ versus $1.5 \pm$ $0.13 \mathrm{~mm}$ ) of COX2KD mice did not differ from control mice (Figure 1, B and $\mathrm{C}$ ). On the other hand, administration of the $\mathrm{PGE}_{2}$ receptor EP4 antagonist ONO-AE3-208
A

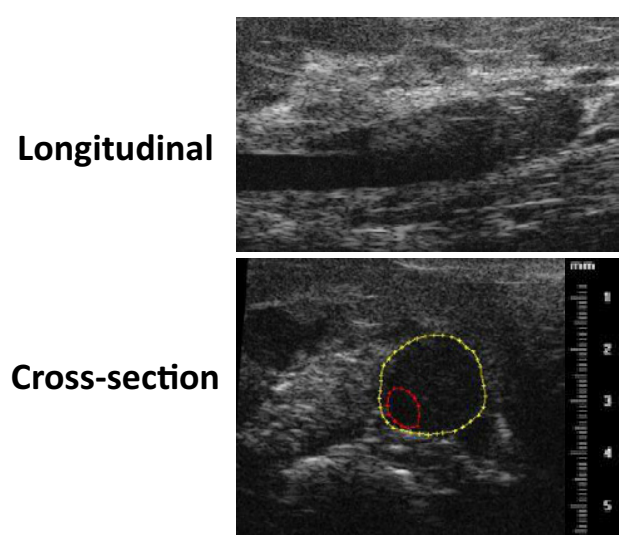

COX2KD
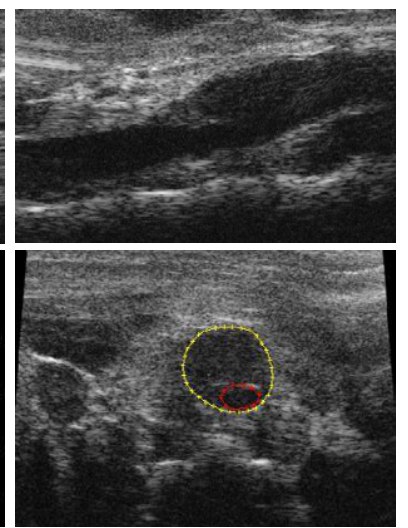

AE3-208
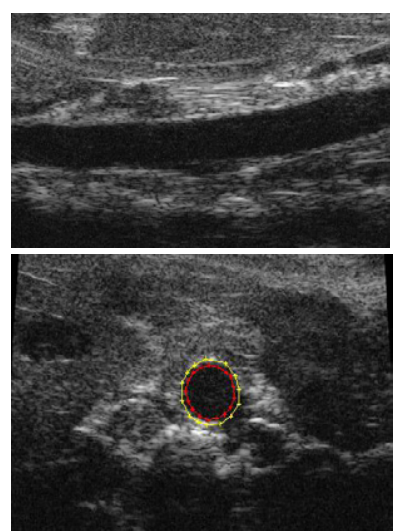

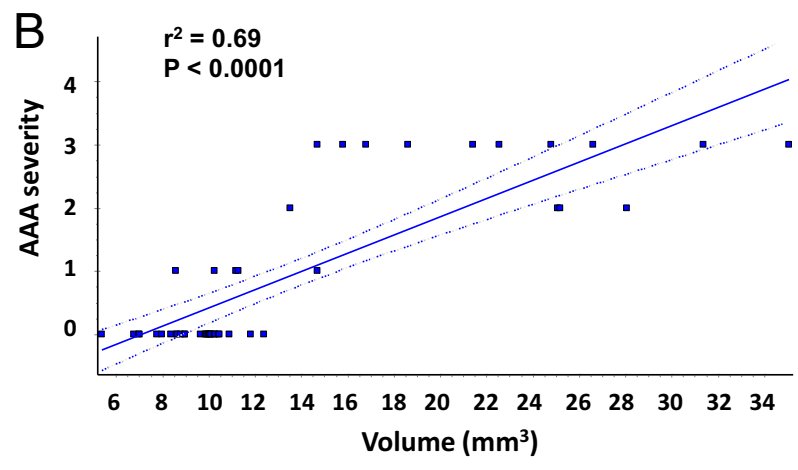

Figure 2. Ultrasonographic detection of AAA. Ultrasonographic imaging is used to monitor the progression of AAA during the 4-week course of AngII infusion. A: Longitudinal and cross-sectional imaging revealed AAAs. In the cross-sectional view, the red outline depicts the lumen; and the yellow outline, the outer wall of the vessel. B: Linear regression analysis reveals good correlation between aortic volume measurements and AAA severity classification $\left(r^{2}=0.69, P<0.0001\right)$. Dotted lines, 95\% CIs. 
via the drinking water resulted in significantly lower AAA incidence [6 (26\%) of $23, P<0.01$ ], with no severe type III aneurysms, and a smaller average abdominal aortic diameter $(1.1 \pm 0.1 \mathrm{~mm}, P<0.05)$ versus control mice. To ensure that the mice were receiving adequate drug via the drinking water, plasma concentrations of drug were evaluated. Drug-treated mice had measurable levels at the 28-day end point (average, $56 \pm 20 \mathrm{ng} / \mathrm{mL} ; n=17$ ), whereas all selected control and COX2KD mouse plasma samples were lower than the lowest limit of detection $(<1$ $\mathrm{ng} / \mathrm{mL}, n=10)$. Representative aortas at the gross dissection level revealed grossly remodeled vessel walls, frequently with thrombus formation, especially in the control and COX-2 genetic knockdown groups (Figure 1D).

\section{Ultrasonographic Imaging of $A A A$}

The development and progression of AAA were followed by two-dimensional B-mode high-resolution ultrasonographic imaging at three intervals (days 7, 14, and 28). We detected most of these AAAs (representative images are in Figure 2A), and the measured diameters at day 28 correlated well with those measured at dissection by micrometer measurements. Thoracic or ascending aortic arch aneurysms were missed by ultrasonographic screening because these areas were not scanned.

Power Doppler measurements were performed to obtain geometric parameters of the AAA for reconstruction into aortic volumes. We found that the aortic volume measurements correlated nicely with the AAA severity score (Figure 2B, $r^{2}=0.69, P<0.0001$ )

\section{Aortic Rupture in Angll-Treated Mice}

Although aortic rupture occurred in close to one third of control animals [ $9(29 \%)$ of 31 ], it was lower in the other two groups [4 (15\%) of 27 COX2KD and 2 (9\%) of 23 EP4 antagonist treated; Figure $3 \mathrm{~A}]$. Based on power analysis, significantly more animals would have to be studied/treated to judge the genetic effects of COX2KD/EP4 antagonist efficacy on rupture rate because of the low incidence (see Discussion). We, however, made some qualitative assessments about the rupture rate. As $w^{27}$ and others ${ }^{10,15,26}$ have previously noted, most of the aortic ruptures occurred within the first week of Angll infusion and they occurred at the suprarenal abdominal aorta region and also at the aortic arch (Figure 3, B and C).

\section{EP4 Receptor Protein Expression Found in Human AAA and Normal Aorta}

Previously, gene expression for the prostaglandin EP4 receptor subtype was confirmed by $\mathrm{RT}$-PCR analysis in human AAA specimens. ${ }^{16}$ We collected 25 AAA specimens from patients undergoing elective/emergency surgical repair, along with three normal aorta sections from the same infrarenal segment (see Supplemental Table S1 at $h t t p: / / a j p . a m j p a t h o l . o r g)$, and examined protein expression for EP4 by using Western blot analysis. A histological assessment revealed the differences in vessel wall structure between the normal and AAA samples (Figure 4, A and B).
A

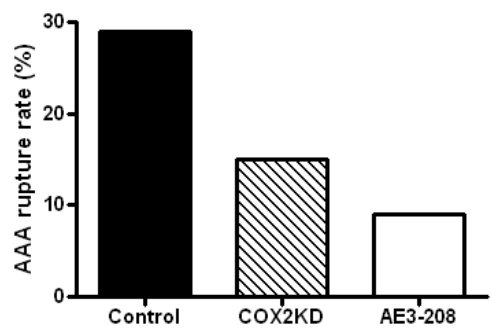

B

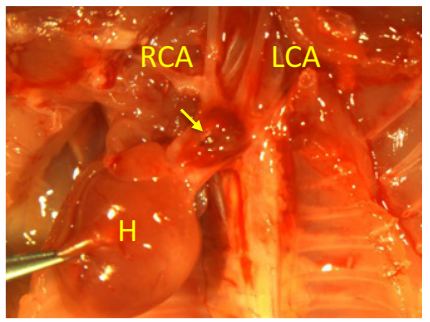

\begin{tabular}{lcccccc} 
C & \multicolumn{6}{c}{ Time and location specificity of aortic rupture } \\
\hline Groups & Week1 & Week2 & Week4 & Abdominal & Aortic arch & Total rupture \\
\hline Control & 7 & 1 & 1 & 6 & 3 & 9 \\
COX2KD & 4 & 0 & 0 & 3 & 1 & 4 \\
AE3-208 & 2 & 0 & 0 & 2 & 0 & 2 \\
\hline
\end{tabular}

Figure 3. Aortic rupture death in AngII-treated mice. A: The rupture rate is lower in COX2KD and EP4 antagonist-treated mice versus the control group ( $n=9, n=$ 4 , and $n=2$, respectively, from $n=31, n=27$, and $n=23$, started in the study in the three groups). The Discussion describes implications of these findings based on power analysis. B: Representative aortic arch rupture in an apoE-deficient mouse treated with AngII. C: In this study, nearly all aortic ruptures occur during the first week of AngII infusion in both abdominal and arch (thoracic) regions. H, heart; LCA, left common carotid artery; RCA, right common carotid artery.

The latter displayed disruptions of the elastic laminae, along with collagen deposition, vascularization, and inflammatory infiltrates, and the intimal layer was often obliterated and replaced by a fibrin-rich nonocclusive intraluminal thrombus. The adventitial layer revealed aneurysmal granulomas with follicle-like aggregates of inflammatory infiltrates in close proximity to vessels of the vasa vasorum. Aortic layers from the control samples were normal in structure. Western blot analysis revealed that a band consistent with EP4 expression (53 kDa) was detected in both AAA and non-AAA tissues (Figure 4C) but not in lipopolysaccharide-challenged leukocytes, in which EP4 receptor expression was suppressed $^{29}$ and COX-2 was induced. From a qualitative assessment, differences in EP4 expression between AAA and non-AAA samples were not apparent and quantitative densitometry analysis between AAA $(n=22)$ and non-AAA $(n=3)$ did not detect significant differences with normalization of EP4 expression to that of $\beta$-actin (Figure 4D). EP4 receptor expression was also detected in Angll-induced aneurysmal tissue of mice (data not shown).

\section{AE3-208 Administration Does Not Affect Global Macrophage Recruitment but Affects the Aortic Inflammatory Milieu}

To begin to understand why the EP4 antagonist attenuated AAA incidence and severity, we examined the re- 

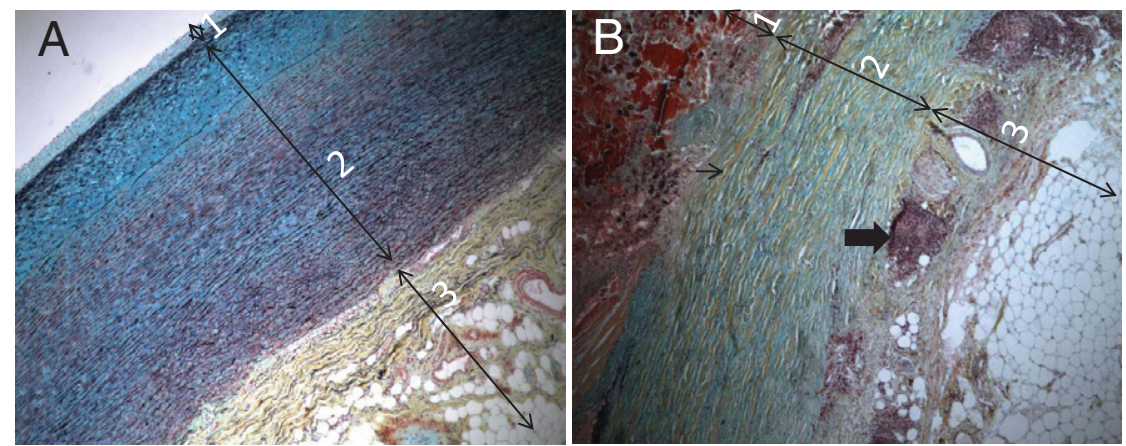

C

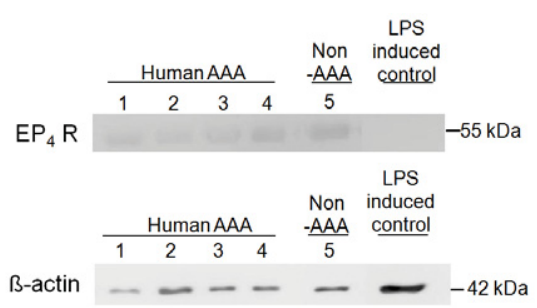

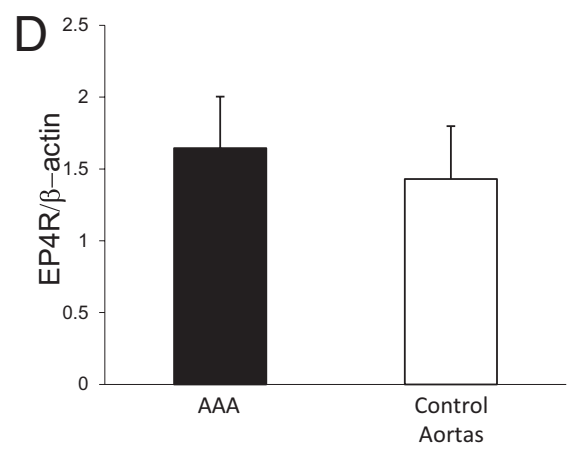

Figure 4. EP4 receptor expression in human normal aorta and AAA specimens. Histological assessment of normal aorta (A) and AAA (B) using Movat's pentachrome stain. Intimal, medial, and adventitial layers are numbered 1,2, and 3, respectively. Thin arrow, collagen deposition in the medial layer of AAA; thick arrow, an aneurysmal granuloma in the adventitia of the AAA. C: Western blot detection of immunoreactive EP4 receptor (the position of the 55$\mathrm{kDa}$ standard marker is shown on the right) in human AAA and normal abdominal aorta (nonAAA). The control sample is protein obtained from lipopolysaccharide (LPS)-stimulated human leukocytes, where EP4 expression is suppressed. ${ }^{28} \beta$-Actin immunoblots serve as loading controls. D: Densitometry analysis of EP4 expression. Ratios of $\mathrm{EP}_{4} \mathrm{R}$ expression are generated by normalization to $\beta$-actin loading control. Normalized EP4 levels are expressed as mean \pm SEM. Expression in AAA $(n=22)$ does not differ significantly from that in non-AAA $(n=3) . P>0.05$. cruitment of macrophages to the aortic wall by assessing expression of a pan-macrophage marker, CD68, using $\mathrm{IHC}$. However, there was no apparent difference among the three groups (Figure 5A) in aortic macrophage numbers. Further examination of macrophage phenotype, characterized as pro-inflammatory (M1), using the marker CD80, versus immunomodulatory/tissue remodeling (M2), with CD163 labeling, ${ }^{30}$ indicated relatively fewer CD80-labeled cells, with enhanced numbers of
CD163 labeling in EP4 antagonist-treated mice compared with control mice (Figure 5, B and C; $P<0.01$ for both CD80 and CD163). By using the pan-T-cell marker, CD90.2, there was a graded reduction in expression in COX2KD and AE-308 samples, with parallel reductions in IL-17 and MIP-1 $\alpha$ (Figure 5, D-F; $P<0.01)$. Moreover, we found attenuated gelatinase activity consistent with MMP-2 and MMP-9 in AE3-208-treated mice compared with control mice (Figure 5G).
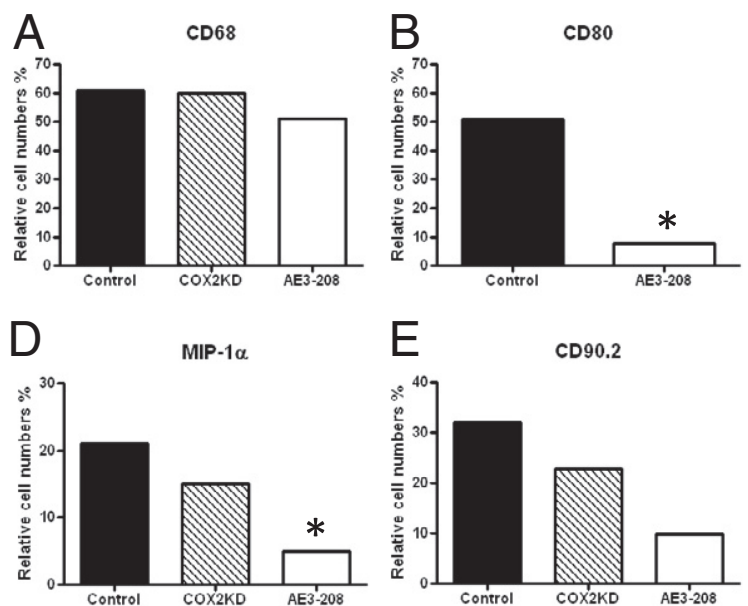

G

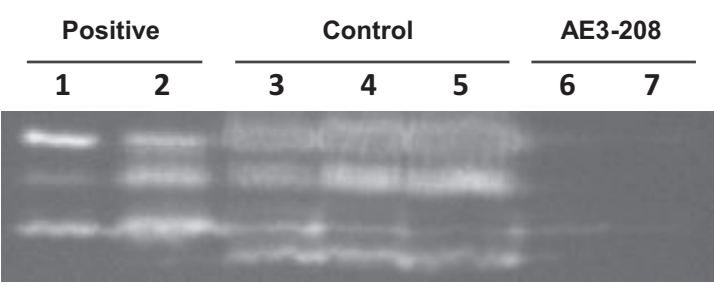

proMMP-9

MMP-9

proMMP-2

MMP-2
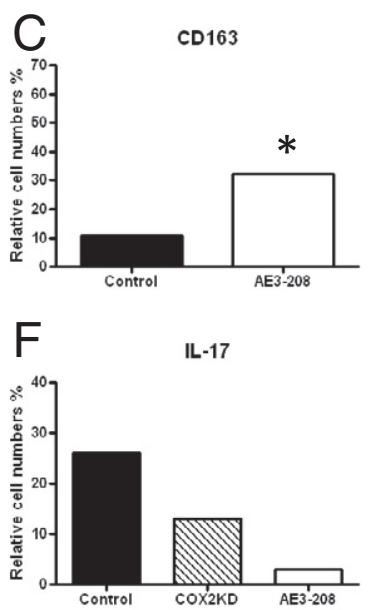

Figure 5. AF3-208 administration reduces the inflammatory phenotype found in aortas of AngIItreated mice. Quantification of global macrophage numbers using CD68 expression (A), pro-inflammatory M1 macrophage marker CD80 (B), immunomodulatory M2 macrophage marker CD163 (C) MIP-1 $\alpha$ (D), pan-T-cell marker CD90.2 (E), and IL-17 via counting positively stained cells relative to DAPI-stained cells and generating a percentage ( $n=5$ to 8 in each group) (F), as described in Materials and Methods. ${ }^{*} P<0.01$ versus control. G: Representative gel of gelatinase activities from a positive mouse embryo fibroblast cell line (lanes 1 and 2), control AngII-treated aortas (lanes 3 to 5), and AE3-208-treated mouse aortas (lanes 6 and 7). Wells are loaded with equal protein amounts, and a gelatin zymography assay is performed as described in Materials and Methods. MMP activities are significantly reduced in AE3-208-treated mouse aortic samples. 
A
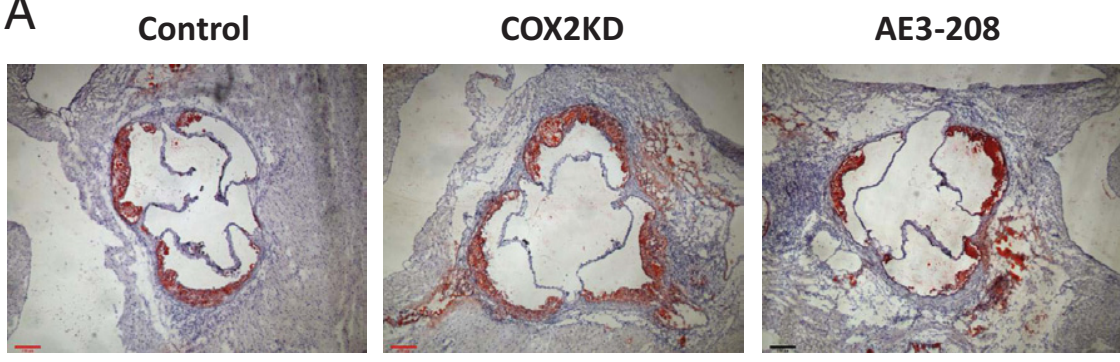

B

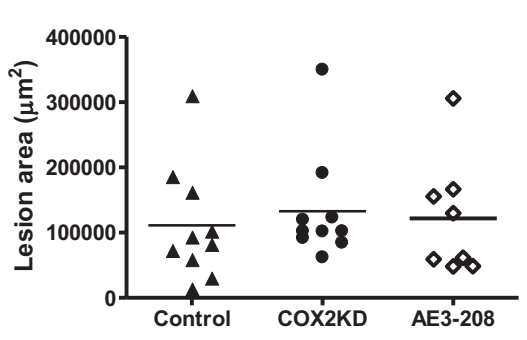

\section{C}

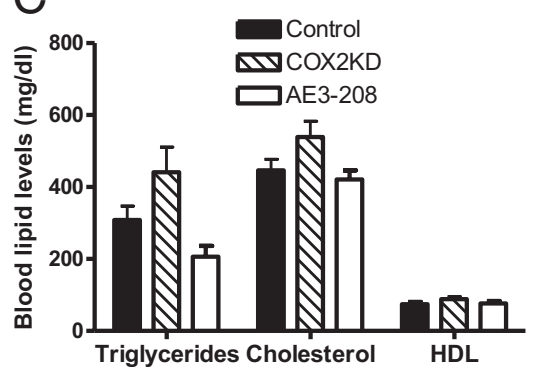

Figure 6. Aortic root atherosclerotic lesions are not affected by either COX2KD or EP4 antagonist treatment. A: Representative oil red Ostained aortic root images of control, COX2KD, and EP4 antagonist-treated mice. B: Quantification of aortic root atherosclerotic lesion area using Image-Pro Plus software $(n=8$ to 11$) . P>$ 0.05 . C: Analysis of triglycerides, total cholesterol, and high-density lipoprotein cholesterol in control $(n=15)$, COX2KD $(n=22)$, and AE3 208 -treated ( $n=13$ ) groups of mice.

\section{COX2KD or EP4 Antagonist Administration Does Not Affect Aortic Root Atherosclerotic Lesions}

To evaluate the impact of genetic COX2KD or pharmacological inhibition of EP4 in atherogenesis on a normal chow diet, mouse hearts from the mice at end point (aged 4 months) were collected and aortic roots were crosssectioned and stained with oil red $\mathrm{O}$ for atherosclerotic lesion quantification. Neither COX2KD nor pharmacological inhibition of EP4 signaling significantly affected lesion size compared with control mice (Figure 6, A and B). Although there were some variations in triglyceride levels between groups, there were no significant differences in plasma total cholesterol or high-density lipoprotein cholesterol when comparing the control and EP4 antagonisttreated mice (Figure 6C).

\section{Discussion}

In the present study, we discovered that pharmacological inhibition of the $\mathrm{PGE}_{2}$ receptor EP4 subtype with AE3-208 significantly decreased AAA incidence/severity, and the inflammatory phenotype in the vessel wall, compared with control male apoE-deficient mice using the Angllinduced AAA model, without influencing atherosclerotic lesion size and overall global macrophage recruitment. Moreover, we also demonstrated expression of the EP4 receptor at the protein level in human and mouse AAAs.

The Angll AAA model is widely used in aortic aneurysm research because certain facets of the model resemble human disease acquisition. ${ }^{22,23}$ Angll is reported to regulate inflammatory COX-2 enzyme expression in various settings, ${ }^{31,32}$ and COX-2 expression has been shown in mouse and human AAAs. ${ }^{8,9}$ The hypothesis that Angll can evoke AAA progression via an inflammatory COX-2 pathway is reasonable given that knockout of the corresponding gene (Ptgs2) in mice or administration of the selective COX-2 inhibitor, celecoxib, attenuates AAA incidence. ${ }^{9,10}$ Subsequent studies ${ }^{15}$ working downstream of COX-2 with mPGES-1 also supported the notion of a COX-2/mPGES-1 connection to AAA pathogenesis. However, the particular receptor subtype for $\mathrm{PGE}_{2}$ that would mediate this action was not previously examined when we undertook this study. $\mathrm{PGE}_{2}$ binds four EP receptor subtypes (EP1 to EP4). ${ }^{33}$ We chose to examine EP4 first because the mRNA for this receptor subtype was previously identified in human AAA specimens and aortic smooth muscle cells. ${ }^{16}$ Our results suggest that there is a COX-2/mPGES-1/PGE 2 -EP4 pathway that participates in the inflammatory progression of AAA in the Angll mouse model. The mechanisms are not entirely clear, but this signaling pathway does not appear to be acting via modulation of global macrophage recruitment. It appears to modulate macrophage phenotype, as assessed by CD80 and CD163 expression (Figure 5), and influence some cytokines/chemokines (IL-17 and MIP$1 \alpha)$ and MMP-2 and MMP-9 (Figure 5).

While we were preparing this article, a recent study by Tang et $\mathrm{al}^{34}$ reported that, in the setting of bone marrow transplantation into irradiated hosts, EP4 deletion on bone marrow-derived cells enhanced inflammation and Angll-induced AAA formation, suggesting that $P G E_{2}$ is acting via an anti-inflammatory pathway. These apparently conflicting results with the combined data described herein, plus the studies of Loftin ${ }^{9,10}$ and FitzGerald ${ }^{15}$ and coworkers, deserve to be compared and contrasted. For instance, the irradiation for bone marrow transplantation resulted in a much lower AAA incidence in their control group of mice $(50 \%)^{34} \mathrm{com}$ pared with that in previous studies (70\% to $90 \%)^{9,15,26}$ and much higher blood lipid levels in their EP4-deleted mice. The studies using COX-2 inhibition/gene deletion, ${ }^{9,10}$ mPGES-1 deletion, ${ }^{15}$ or EP4 pharmacological inhibition (Figure 6) did not show significant lipid profile 
differences compared with the control mice. These unanticipated changes in lipid levels in the bone marrowreconstituted mice led to enhanced lipid deposits on the thoracic aorta of their EP4 ${ }^{-/-} /$low-density lipoprotein receptor $^{-1-}$ mice (much more than their control mice) ${ }^{34}$ that were not observed in EP4 antagonist-treated mice (Figure 6). The loss of one EP receptor subtype likely led to the compensatory up-regulation and/or signaling via other EP receptor subtypes in the bone marrow reconstitution experiments. EP receptors are notorious for their opposite signaling cascades, with EP2 and EP4 increasing CAMP production and EP3 leading to decreased CAMP. ${ }^{33}$ This compensatory mechanism may have come into play in the EP4 bone marrow-deleted mice but not in mice treated with AE3-208. Because IL-17 was reduced in EP4 antagonist-treated mouse aortas (Figure 5), another potential explanation for variable results is that IL-17 can be both pro-inflammatory and anti-inflammatory, depending on the specific context and disease setting, ${ }^{20,35,36}$ and IL-17 signaling could have been affected differentially in the studies. We speculate that IL-17 may play a part of the role in the regulation of the inflammatory process in AAA formation because PGE $\mathrm{E}_{2}$-EP4 activation could induce inflammatory cytokine $\mathrm{IL}-17$ production, ${ }^{20,21}$ which could potentially trigger macrophage phenotypic changes and also affect chemokine expression (eg, MIP-1 $\alpha$ ). This could further exacerbate inflammatory processes and lead to MMP activation ${ }^{17,19}$ in AAA pathogenesis. Each of these conjectures will require further experimentation in future studies.

In our studies herein, we also tested mice with knocked-down COX-2 expression. These mice differ from COX-2-knockout mice because they retain detectable levels of COX-2 expression (10\% to $30 \%$ ), depending on the tissue, and do not display significant differences from control mice in urinary metabolites of $\mathrm{PGE}_{2}{ }^{24}$ Surprisingly, COX-2 genetic knockdown mice did not differ substantially from the control group in our studies in terms of AAA incidence and severity compared with the strongly significant differences previously described with selective COX-2 inhibition or COX-2 knockout. ${ }^{9,10}$ Perhaps the residual COX-2 expression in COX-2 genetic knockdown mice was sufficient to generate enough $\mathrm{PGE}_{2}$ to still signal via EP4, or there were COX-1 compensatory changes in the aorta. Previously, we had generated COX-1-knockdown mice and demonstrated that only $10 \%$ to $20 \%$ COX-1 expression and prostaglandin $\mathrm{F}_{2 \alpha}$ production in the ovaries/uterus were sufficient to rescue the parturition delay that was present in COX-1-knockout mice. ${ }^{37}$

In this study, the aortic rupture rate was $29 \%$ in the control group of animals, which is consistent with previous findings. The rate was reduced to $9 \%$ in EP4 antagonist-treated mice. Because of the fairly low event rate, we would need approximately 100 mice in each group to achieve significance at the 0.05 level, with $80 \%$ power to evaluate AE3-208 for its efficacy in reducing aortic ruptures. Therefore, the results on reduction of rupture rate in AE3-208-treated mice could be considered as hypothesis generating because the current sample size would only have $45 \%$ power to detect the observed difference based on the power calculation. There are no clinically used pharmacological treatments that reduce aortic rupture and progression, and perhaps modulating the EP4 receptor could be relevant in this respect. We have been able to follow the aortic dissection event, which precedes rupture in this model, and the aortic wall remodeling and 3D geometry (Figure 2) by ultrasonographic imaging in mice. ${ }^{27}$ Interestingly, the aortic ruptures that do occur are not only in the abdominal area but also in the arch (Figure 3). Ascending thoracic aortic aneurysms have been recently noted in the Angll model ${ }^{38}$; however, this area is not readily amenable to imaging compared with the abdominal aorta for 3D measurements. The 3D geometry (volume) showed a significant correlation with the postmortem AAA classification (Figure 2).

In conclusion, this study demonstrated that the $\mathrm{PGE}_{2}$ EP4 signaling pathway contributed to AAA formation. Treatment with an EP4 antagonist significantly reduced inflammation in aneurysmal tissue by modulating the inflammatory phenotype and decreased AAA incidence and severity compared with nontreated control mice. Therefore, pharmaceutical interruption to block the inflammatory process through the $\mathrm{PGE}_{2}$-EP4 signaling pathway may prevent the progression of AAAs.

\section{Acknowledgments}

We thank Dr. Shigeru Matsumoto and Naoya Matumura (Ono Pharmaceutical Co., Ltd.) for coordinating and analyzing the drug plasma concentration of the EP4 antagonist ONO-AE3-208, Janet Creasy for organizing the human aorta collections, and Rob Evers and Lilly Jia for providing their generous technical support.

\section{References}

1. Upchurch GR Jr, Schaub TA: Abdominal aortic aneurysm. Am Fam Physician 2006, 73:1198-1204

2. Lloyd-Jones D, Adams RJ, Brown TM, Carnethon M, Dai S, De Simone G, Ferguson TB, Ford E, Furie K, Gillespie C, Go A, Greenlund K, Haase N, Hailpern S, Ho PM, Howard V, Kissela B, Kittner S, Lackland D, Lisabeth L, Marelli A, McDermott MM, Meigs J, Mozaffarian D, Mussolino M, Nichol G, Roger VL, Rosamond W, Sacco R, Sorlie P, Thom T, Wasserthiel-Smoller S, Wong ND, Wylie-Rosett J: Heart disease and stroke statistics-2010 update: a report from the American Heart Association. Circulation 2010, 121:e46-e215

3. Golledge J, Muller J, Daugherty A, Norman P: Abdominal aortic aneurysm: pathogenesis and implications for management. Arterioscler Thromb Vasc Biol 2006, 26:2605-2613

4. van Vlijmen-van Keulen CJ, Pals G, Rauwerda JA: Familial abdominal aortic aneurysm: a systematic review of a genetic background. Eur $\mathrm{J}$ Vasc Endovasc Surg 2002, 24:105-116

5. McCormick ML, Gavrila D, Weintraub NL: Role of oxidative stress in the pathogenesis of abdominal aortic aneurysms. Arterioscler Thromb Vasc Biol 2007, 27:461-469

6. Thompson RW: Reflections on the pathogenesis of abdominal aortic aneurysms. Cardiovasc Surg 2002, 10:389-394

7. Baxter BT, Terrin MC, Dalman RL: Medical management of small abdominal aortic aneurysms. Circulation 2008, 117:1883-1889

8. Holmes DR, Wester W, Thompson RW, Reilly JM: Prostaglandin E2 synthesis and cyclooxygenase expression in abdominal aortic aneurysms. J Vasc Surg 1997, 25:810-815

9. King VL, Trivedi DB, Gitlin JM, Loftin CD: Selective cyclooxygenase-2 inhibition with celecoxib decreases angiotensin II-induced abdomina aortic aneurysm formation in mice. Arterioscler Thromb Vasc Biol 2006, 26:1137-1143 
10. Gitlin JM, Trivedi DB, Langenbach R, Loftin CD: Genetic deficiency of cyclooxygenase-2 attenuates abdominal aortic aneurysm formation in mice. Cardiovasc Res 2007, 73:227-236

11. Morham SG, Langenbach R, Loftin CD, Tiano HF, Vouloumanos N, Jennette JC, Mahler JF, Kluckman KD, Ledford A, Lee CA, Smithies $\mathrm{O}$ : Prostaglandin synthase 2 gene disruption causes severe renal pathology in the mouse. Cell 1995, 83:473-482

12. Dinchuk JE, Car BD, Focht RJ, Johnston JJ, Jaffee BD, Covington MB, Contel NR, Eng VM, Collins RJ, Czerniak PM, Gorry SA, Trzaskos JM: Renal abnormalities and an altered inflammatory response in mice lacking cyclooxygenase II. Nature 1995, 378:406-409

13. Fitzgerald GA: Coxibs and cardiovascular disease. N Engl J Med 2004, 351:1709-1711

14. Grosser T, Fries S, FitzGerald GA: Biological basis for the cardiovascular consequences of COX-2 inhibition: therapeutic challenges and opportunities. J Clin Invest 2006, 116:4-15

15. Wang M, Lee E, Song W, Ricciotti E, Rader DJ, Lawson JA, Pure E, FitzGerald GA: Microsomal prostaglandin E synthase-1 deletion suppresses oxidative stress and angiotensin II-induced abdominal aortic aneurysm formation. Circulation 2008, 117:1302-1309

16. Bayston T, Ramessur S, Reise J, Jones KG, Powell JT: Prostaglandin E2 receptors in abdominal aortic aneurysm and human aortic smooth muscle cells. J Vasc Surg 2003, 38:354-359

17. Wang $Y$, Ait-Oufella $H$, Herbin $O$, Bonnin $P$, Ramkhelawon B, Taleb S, Huang J, Offenstadt G, Combadiere C, Renia L, Johnson JL, Tharaux PL, Tedgui A, Mallat Z: TGF-beta activity protects against inflammatory aortic aneurysm progression and complications in angiotensin II-infused mice. J Clin Invest 2010, 120:422-432

18. Tieu BC, Lee C, Sun H, Lejeune W, Recinos A 3rd, Ju X, Spratt H, Guo DC, Milewicz D, Tilton RG, Brasier AR: An adventitial IL-6/MCP1 amplification loop accelerates macrophage-mediated vascular inflammation leading to aortic dissection in mice. J Clin Invest 2009, 119:3637-3651

19. Shimizu K, Shichiri M, Libby P, Lee RT, Mitchell RN: Th2-predominant inflammation and blockade of IFN-gamma signaling induce aneurysms in allografted aortas. J Clin Invest 2004, 114:300-308

20. Yao C, Sakata D, Esaki Y, Li Y, Matsuoka T, Kuroiwa K, Sugimoto Y, Narumiya S: Prostaglandin E2-EP4 signaling promotes immune inflammation through Th1 cell differentiation and Th17 cell expansion. Nat Med 2009, 15:633-640

21. Sheibanie AF, Yen JH, Khayrullina T, Emig F, Zhang M, Tuma R, Ganea D: The proinflammatory effect of prostaglandin E2 in experimental inflammatory bowel disease is mediated through the IL23 $\rightarrow$ LL-17 axis. J Immunol 2007, 178:8138-8147

22. Daugherty A, Cassis LA: Mouse models of abdominal aortic aneurysms. Arterioscler Thromb Vasc Biol 2004, 24:429-434

23. Daugherty A, Manning MW, Cassis LA: Angiotensin II promotes atherosclerotic lesions and aneurysms in apolipoprotein E-deficient mice. J Clin Invest 2000, 105:1605-1612
24. Seta F, Chung AD, Turner PV, Mewburn JD, Yu Y, Funk CD: Renal and cardiovascular characterization of COX-2 knockdown mice. Am J Physiol Regul Integr Comp Physiol 2009, 296:R1751-R1760

25. Cao RY, Adams MA, Habenicht AJ, Funk CD: Angiotensin II-induced abdominal aortic aneurysm occurs independently of the 5-lipoxygenase pathway in apolipoprotein E-deficient mice. Prostaglandins Other Lipid Mediat 2007, 84:34-42

26. Daugherty A, Manning MW, Cassis LA: Antagonism of AT2 receptors augments angiotensin II-induced abdominal aortic aneurysms and atherosclerosis. Br J Pharmacol 2001, 134:865-870

27. Cao RY, Amand T, Ford MD, Piomelli U, Funk CD: The murine angiotensin II-induced abdominal aortic aneurysm model: rupture risk and inflammatory progression patterns. Front Pharmacol 2010, 1:1-9

28. Rosner, B: Fundamentals of Biostatistics. ed 7. Belmont, CA, Duxbury Press, 2011

29. Ikegami R, Sugimoto Y, Segi E, Katsuyama M, Karahashi H, Amano F, Maruyama T, Yamane H, Tsuchiya S, Ichikawa A: The expression of prostaglandin E receptors EP2 and EP4 and their different regulation by lipopolysaccharide in $\mathrm{C} 3 \mathrm{H} / \mathrm{HeN}$ peritoneal macrophages. J Immunol 2001, 166:4689-4696

30. Badylak SF, Valentin JE, Ravindra AK, McCabe GP, Stewart-Akers AM: Macrophage phenotype as a determinant of biologic scaffold remodeling. Tissue Eng Part A 2008, 14:1835-1842

31. Tani T, Ayuzawa R, Takagi T, Kanehira T, Maurya DK, Tamura M: Angiotensin II bi-directionally regulates cyclooxygenase-2 expression in intestinal epithelial cells. Mol Cell Biochem 2008, 315:185-193

32. Hu ZW, Kerb R, Shi XY, Wei-Lavery T, Hoffman BB: Angiotensin II increases expression of cyclooxygenase-2: implications for the function of vascular smooth muscle cells. J Pharmacol Exp Ther 2002 303:563-573

33. Sugimoto Y, Narumiya S: Prostaglandin E receptors. J Biol Chem 2007, 282:11613-11617

34. Tang EH, Shvartz E, Shimizu K, Rocha VZ, Zheng C, Fukuda D, Shi GP, Sukhova G, Libby P: Deletion of EP4 on bone marrow-derived cells enhances inflammation and angiotensin II-induced abdominal aortic aneurysm formation. Arterioscler Thromb Vasc Biol 2011, 31: 261-269

35. Esaki Y, Li Y, Sakata D, Yao C, Segi-Nishida E, Matsuoka T, Fukuda K, Narumiya S: Dual roles of PGE2-EP4 signaling in mouse experimental autoimmune encephalomyelitis. Proc Natl Acad Sci U S A 2010, 107:12233-12238

36. Chen S, Crother TR, Arditi M: Emerging role of IL-17 in atherosclerosis. J Innate Immun 2010, 2:325-333

37. Yu Y, Cheng Y, Fan J, Chen XS, Klein-Szanto A, Fitzgerald GA, Funk CD: Differential impact of prostaglandin $\mathrm{H}$ synthase 1 knockdown on platelets and parturition. J Clin Invest 2005, 115:986-995

38. Rateri DL, Moorleghen JJ, Balakrishnan A, Owens AP 3rd, Howatt DA, Subramanian V, Poduri A, Charnigo R, Cassis LA, Daugherty A: Endothelial cell-specific deficiency of Ang II type 1a receptors attenuates Ang II-induced ascending aortic aneurysms in LDL receptor-/- mice. Circ Res 2011, 108:574-581 